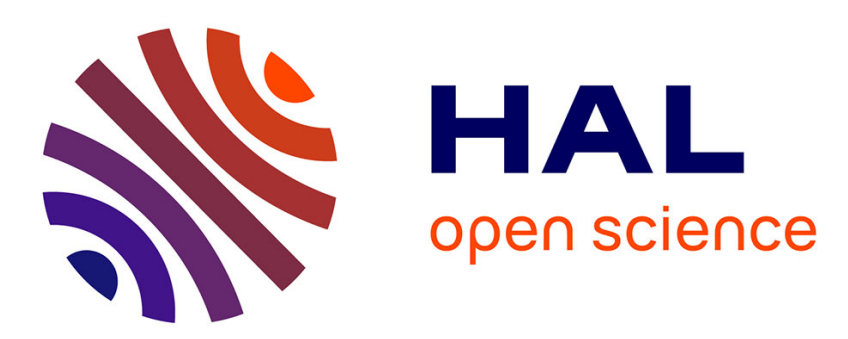

\title{
Gaze perception induces early attention orienting effects in occipito-parietal regions
}

José Luis Ulloa, Stéphanie Dubal, Lydia Yahia-Cherif, Nathalie George

\section{To cite this version:}

José Luis Ulloa, Stéphanie Dubal, Lydia Yahia-Cherif, Nathalie George. Gaze perception induces early attention orienting effects in occipito-parietal regions. Neuropsychologia, 2018, 109, pp.173-180. 10.1016/j.neuropsychologia.2017.12.029 . hal-01700862

\section{HAL Id: hal-01700862 https://hal.sorbonne-universite.fr/hal-01700862}

Submitted on 5 Feb 2018

HAL is a multi-disciplinary open access archive for the deposit and dissemination of scientific research documents, whether they are published or not. The documents may come from teaching and research institutions in France or abroad, or from public or private research centers.
L'archive ouverte pluridisciplinaire HAL, est destinée au dépôt et à la diffusion de documents scientifiques de niveau recherche, publiés ou non, émanant des établissements d'enseignement et de recherche français ou étrangers, des laboratoires publics ou privés. 


\title{
Gaze perception induces early attention orienting effects in occipito-parietal regions ${ }^{\text {is }}$
}

\author{
José Luis Ulloa $^{\mathrm{a}, \mathrm{c}, *}$, Stéphanie Dubal ${ }^{\mathrm{a}}$, Lydia Yahia-Cherif ${ }^{\mathrm{b}}$, Nathalie George ${ }^{\mathrm{a}, \mathrm{b}}$ \\ a Inserm U 1127, CNRS UMR 7225, Sorbonne Universités, UPMC Univ Paris 06 UMR S 1127, Institut du Cerveau et de la Moelle épinière (ICM), Social and Affective \\ Neuroscience (SAN) Laboratory and Centre MEG-EEG, 75013 Paris, France \\ ${ }^{\mathrm{b}}$ Inserm U 1127, CNRS UMR 7225, Sorbonne Universités, UPMC Univ Paris 06 UMR S 1127, Institut du Cerveau et de la Moelle épinière (ICM), ENS, Centre MEG-EEG, \\ 75013 Paris, France \\ ${ }^{\mathrm{c}}$ Millennium Nucleus Biology of Neuropsychiatric Disorders Nu-MIND, Facultad de Ciencias, Universidad de Valparaíso, Chile
}

Keywords:

Eye gaze

Social attention

Attention orienting

Magnetoencephalography

\begin{abstract}
A B S T R A C T
Other's eye gaze is a powerful attention orienting cue that can change our perception of objects in the environment. Here, we seek to characterize the influence of attention orienting by eye gaze on the neural processing of visual targets. We used a Posner-like cueing paradigm to investigate with magnetoencephalography the brain responses associated with target processing. We analyzed the cerebral sources of the evoked responses to visual targets that were validly or invalidly cued by eye gaze. The effect of attention orienting was reflected in faster reaction times to valid than invalid targets. At the brain level, we showed an early influence of attention orienting by gaze with enhanced brain responses for invalid relative to valid targets. This influence was maximum contra-laterally to the target, with a right hemisphere dominance. Responses to targets presented in the left visual field were modulated between 91 and $400 \mathrm{~ms}$ in the right posterior parietal and occipital cortices. Responses to targets presented in the right visual field were modulated between 174 and $218 \mathrm{~ms}$ in the left superior parietal cortex. Our results confirm previous EEG studies that demonstrated early influence of attention orienting by gaze on target processing and provide evidence for the sources of this effect in occipito-parietal regions. This early influence may reflect the first stage of the perceptual changes induced by social attention.
\end{abstract}

\section{Introduction}

Our daily perceptual experience is embedded in a rich social environment for which we have developed exquisite cognitive abilities. We are not only able to efficiently detect and respond to other's social signals such as eye gaze and emotional expressions, these signals also tend to change the way we perceive surrounding objects. For example, other's gaze typically elicits attention orienting toward gazed-at objects (e.g. Driver et al., 1999) and can impinge new and powerful meanings to events or objects in the environment (e.g. Becchio et al., 2008; Bayliss et al., 2006). In Posner-like paradigms, averted gaze is used as a central attentional cue, followed by a peripheral target that the subject has to detect, discriminate, categorize, or identify. The attention orienting effect associated with averted gaze perception is reflected in faster responses to gazed-at (or valid) targets than to targets appearing on the opposite side of the screen relative to the gazed-at location (or invalid targets; e.g. Friesen and Kingstone, 1998; Driver et al., 1999). This gaze cueing effect is typically observed for cue-target asynchrony between 100 and $700 \mathrm{~ms}$, with little if any inhibition of return (for review, Frischen et al., 2007). This confers to attention orienting processes induced by gaze properties that are reminiscent of both endogenous and exogenous attention, which has raised questions regarding their cerebral underpinnings. Functional magnetic resonance imaging (fMRI) and electroencephalography (EEG) studies have provided insight regarding the brain regions and the temporal dynamics associated with the attention orienting effects of perceiving other's eye gaze (see Nummenmaa and Calder, 2009; Itier and Batty, 2009 for reviews). However, there is still a lack of evidence for the early recruitment of visuospatial cortices in attention orienting by gaze.

In a series of event-related potential (ERP) studies, Schuller and Rossion (2001, 2004, 2005) demonstrated the early influence (in the $100 \mathrm{~ms}$ range) of attention orienting to eye gaze on the neural

\footnotetext{
This research was conducted in the SAN team and in the MEG-EEG Centre of the Institut du Cerveau et de la Moelle Epinière (ICM).

* Correspondence to: Social and Affective Neuroscience team, ICM, UPMC/CNRS/INSERM, Institut du Cerveau et de la Moelle Epinière (ICM), GHU Pitié-Salpêtrière, 47, Bd de l'Hôpital,l'Hôpital, F-75651 Paris Cedex 13, France.

E-mail addresses: joseluisulloafulgeri@gmail.com (J.L. Ulloa), stephanie.dubal@upmc.fr (S. Dubal), lydia.yahia-cherif@upmc.fr (L. Yahia-Cherif), nathalie.george@upmc.fr (N. George).
} 
processing of visual targets. The studies showed enhanced P1 amplitude in response to valid compared to invalid targets, and enhanced P3 amplitude in response to invalid compared to valid targets. This early influence has been confirmed by other ERP studies that compared directional versus non directional targets (Hietanen et al., 2008), or gaze versus symbolic cues such as arrows (Tipper et al., 2008; Brignani et al., 2009). However, these studies did not examine the cerebral sources responsible for these effects. Brain imaging techniques with high spatial resolution such as fMRI have shown activations of occipito-parietal, temporal, and frontal cortices in response to eye gaze cues and ensuing attention orienting processes (Hietanen et al., 2006; Tipper et al., 2008; Sato et al., 2009, for a review see Nummenmaa and Calder, 2009). However, given the low temporal resolution of this method, some of these activations may reflect eye gaze processing while others may reflect the modulation of target processing induced by eye gaze. Magnetoencephalography (MEG) can allow us to investigate with precise temporal resolution the cerebral substrates of visual target processing and how it is modulated by attention orienting to gaze. So far, only a few MEG studies have addressed this question. The study of Lachat et al. (2012b) used a Posner-like paradigm to investigate the impact of emotion on attention cueing by gaze. Central, neutral faces turned either fearful or happy while their eye gaze simultaneously shifted toward the right or the left of the screen. This emotional attention cue was followed by the appearance of target checkerboard, either on the gazedat (valid) or on the not gazed-at (invalid) side of the screen. This study demonstrated an early (between 55 and $70 \mathrm{~ms}$ ) differential recruitment of the left superior parietal lobe and left middle occipital gyrus in response to valid versus invalid targets cued by fearful-as opposed to happy—gaze. Another MEG study examined attention cueing by gaze, this time using neutral faces. Nagata et al. (2012) used a Posner-like paradigm to examine attention orienting induced by central, averted eye gaze and peripheral cues. They observed only a relatively late event-related field (ERF) response (later than $250 \mathrm{~ms}$ ) to the invalid versus valid targets that followed the gaze cues and this effect involved the anterior cingulate cortex. Although other studies have shown early recruitment of occipito-parietal networks for visuospatial attention processes (e.g. Foxe et al., 2003, see also Lachat et al., 2012b), no such evidence was found in Nagata's et al. (2012) study. Finally, Bayless et al. (2013) performed a similar study in children, but did not show any significant difference in brain activations in response to targets associated with the attention cueing by gaze.

The present study aimed at further investigating the attention orienting effect of gaze on the visual processing of surrounding objects. We recorded neuromagnetic responses with MEG in a Posner-like paradigm using alpha-numeric characters as target stimuli and a letter/ symbol categorization task. We based our paradigm on previous studies on eye gaze cueing. Previous reports have shown maximal cueing effect for cue-target asynchrony comprised between 300 and $700 \mathrm{~ms}$ (Friesen and Kingstone, 1998; Driver et al., 1999). Following Schuller and Rossion (2001) who first demonstrated early gaze cueing effects on target processing with EEG and other studies that demonstrated robust gaze cueing effects (e.g. Bayliss et al., 2006), we chose to use a cuetarget asynchrony of $500 \mathrm{~ms}$. The paradigm was also validated in a previous behavioral study of our group (Ulloa et al., 2014). Based on previous EEG and fMRI evidence, we expected to find early modulation of brain responses to targets within occipital and parietal regions following attention orienting by eye gaze.

\section{Methods and materials}

\subsection{Participants}

Twenty-six right-handed healthy volunteers participated in the study (17 female; age range $=19-35$ years, mean age $=23$ years). All participants had normal or corrected-to-normal vision and were naive to the purpose of the experience. All the participants gave written informed consent to participate and received money compensation for their participation in the study. The protocol was approved by the local ethics committee (Comité de Protection des Personnes Ile-de-France VI). Two participants were excluded from data analysis (one was an outlier in the behavioral data and for the other there were technical problems during the MEG recording).

\subsection{Stimuli and task}

The experiment consisted in a Posner-like gaze cueing paradigm (Posner, 1980). Color images of faces served as the cue stimuli. The photographs of 9 male and 9 female neutral faces with direct and averted gaze constituted the stimulus set. All faces subtended a visual angle of $\sim 6.5^{\circ}$ horizontally and $\sim 7.5^{\circ}$ vertically, corresponding to a size of $252 \times 300$ pixels (width $\times$ height). Color images of alphanumeric items served as the target stimuli. They were constituted by 16 symbol and 16 arabic-alphabetic items. These items subtended a visual angle of $\sim 2^{\circ}$ horizontally and $\sim 2^{\circ}$ vertically, corresponding to a size of 40-92 $\times$ 40-70 pixels (width $\times$ height).

The protocol consisted of 6 blocks of attention cueing by eye gaze where the participants performed a target categorization task. Each block comprised 64 trials, resulting in a total of 384 trials. Each trial began with a central fixation cross displayed for $617 \mathrm{~ms}$. The participant was required to fixate on this cross and not to move his/her eyes throughout the trial. A central neutral face with direct gaze replaced the fixation cross. The location of the fixation point remained midway between the eyes. After $1017 \mathrm{~ms}$, the same face was presented but with its eyes shifted to the right or to the left, thus eliciting an apparent motion of eye gaze. After another $517 \mathrm{~ms}$, a target item was displayed laterally at $7^{\circ}$ of visual angle from the centre of the screen; the target item was displayed either on the side cued by the gaze (valid condition) or on the opposite side (invalid condition; $50 \%$ of trials under each condition). Participants were told that eye gaze direction was uninformative of target side. Target stimuli could appear in one of 4 colors: red, blue, yellow, or green. However, each participant viewed only two color versions of each target. One color consistently appeared in the valid condition, while the other consistently appeared in the invalid condition. The association between color and validity was randomized across targets and counterbalanced across participants. The two versions of every target appeared once in each block. This color manipulation was not relevant for the aim of the present study and is only described here for completeness. The participants performed a categorization task; they had to indicate as rapidly and as accurately as possible the category (symbol or letter) of the target by pressing one of two buttons with the index or middle finger of their right hand. The target and the face gaze cue stimuli remained on screen until the participant's response or until $2500 \mathrm{~ms}$ had elapsed. The participant's response was followed by a feedback (thumbs-up or thumbs-down pictures for correct and incorrect responses, respectively) for $300 \mathrm{~ms}$ and after a randomly jittered inter-trial-interval (ITI) of 1500-2500 ms, another trial began (Fig. 1). The association between face cues and

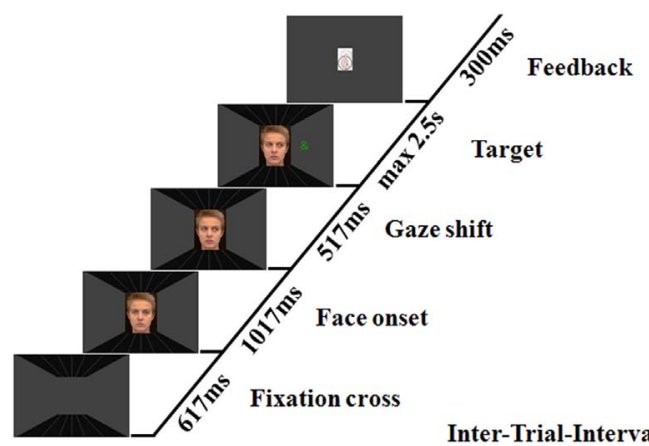

Inter-Trial-Interval: $1.5-2.5 \mathrm{~s}$

Fig. 1. Time course of an example trial. 
target items and the order of target presentation were randomized and counterbalanced across blocks and subjects, with the only constraint that the same target item (under its two different colors) could not be displayed twice in a row. Each block lasted about $5.5 \mathrm{~min}$ and subjects were allowed to rest and stretch a little bit between the blocks while remaining in the MEG system.

At the end of the recording session, two additional blocks of attention cueing by eye gaze were performed where the participants performed the same categorization task followed by an affective rating task. This task was used to assess other research questions that will not be described here.

\section{3. $M E G$ data acquisition}

The experiment was conducted at the MEG-EEG centre of the Centre de Neuroimagerie de Recherche (CENIR), Paris, France. Participants were seated in a dimly lit, sound-proof, magnetically shielded room, with a screen display (resolution of $1400 \times 1050$ pixels, window size $30 \times 18 \mathrm{~cm}$, refresh rate $60 \mathrm{~Hz}$ ) placed at a viewing distance of $85 \mathrm{~cm}$. Stimuli were delivered by a PC computer using a homemade software. They were backprojected onto the screen through a video projector (Panasonic PT-D10000) housed outside the magnetically shielded room and one mirror inside the room. Neuromagnetic fields were recorded with a 306-channels whole-head Elekta Neuromag Triux MEG system comprising 204 orthogonally oriented planar gradiometers and 102 magnetometers regularly distributed at 102 locations over the scalp. Magnetic signals were recorded continuously with a sampling rate of $1 \mathrm{kHz}$ and a low-pass filter set at $330 \mathrm{~Hz}$. In order to control for head movements, head position inside the MEG dewar was checked at the beginning of each block and the subject was asked to place his/her head in the same position within the helmet for every block and to adjust it if necessary (viz. if movement on any of the three directions of the frame exceeded $5 \mathrm{~mm}$ ). Four head position indicator (HPI) coils were affixed to the subject's head. The positions of the HPI coils as well as those of multiple points on the scalp were recorded with a magnetic digitizer (Polhemus Fastrak) in a head coordinate frame defined by three anatomical landmarks constituted by the nasion and the left and right auricular points. Eye movements (electrooculograms, EOG) were recorded by bipolar electrodes, with two electrodes placed at the outher canthi of the eyes for the horizontal eye movements, and two electrodes placed above and below the right eye for the vertical eye movements. Electrocardiogram was monitored by two silver chloride electrodes placed on the left belly and the right collarbone. The recording also included the signal of a photodiode that detected the actual appearance of the stimuli on the screen within the MEG room. This allowed correcting for the delay introduced by the video projector $(30 \mathrm{~ms})$ and averaging event-related magnetic fields (ERFs) precisely time-locked on the actual onset of the target stimuli.

\subsection{MEG data preprocessing}

The preprocessing of MEG data was done for each subject individually using Elekta software and home-made programs implemented in Linux environment. First, the signal-space separation method implemented in MaxFilter version 2.2.1 (Elekta Neuromag) was used to realign head position on the mean position recorded across blocks (in order to correct for any remaining head position change across blocks) and to remove external noise from MEG data. This was complemented by a principal component analysis (PCA) procedure to get rid of remnant noise. Visual inspection of the data allowed us to determine the exclusion of the three first PCA components for both magnetometers and gradiometers. Next, MEG data were visually inspected to exclude the epochs containing eye blinks, eye movements, muscle activity, or other artifacts. We did not apply any cardiac correction; cardiac artifacts were indeed still visible in PCA-corrected data but they were not time-locked to the events of the protocol and visual examination showed that their amplitude was greatly reduced in the ERF averages, so that no contamination of averaged evoked magnetic fields by the cardiac activity was visible.

\subsection{ERF data average}

ERFs were computed by averaging the artifact-free MEG data segments time locked to $1635 \mathrm{~ms}$ before (encompassing the time intervals of the fixation cross display, the initial face and the eye gaze cue presentation) and $700 \mathrm{~ms}$ after the appearance of the target separately for each condition of interest, across the 6 initial blocks of the experiment. Only the trials where a correct categorization response was given were retained. We computed averaged ERFs separately for valid and invalid targets displayed on the right and on the left sides of the screen. All ERF averages were baseline corrected according to the $100 \mathrm{~ms}$ preceding the onset of the initial face-which corresponded to the time interval between 1635 and $1535 \mathrm{~ms}$ before the target display-and digitally lowpass filtered at $40 \mathrm{~Hz}$. The mean number of trials averaged ( \pm SD) were 75 ( \pm 10 ), 74 ( \pm 12 ), 74 ( \pm 10 ) and 75 ( \pm 12 ) for the invalid left, invalid right, valid left and valid right target conditions respectively. There was no statistical difference in the number of trials averaged across conditions $(\mathrm{F}<1)$.

\subsection{Source localization}

Cortical current source density mapping was obtained using a distributed source model in each subject and condition. We used Brainstorm software (http://neuroimage.usc.edu) to perform cortical current source density mapping from the MEG time series at each time point in each condition and for each participant by means of a linear inverse estimator, the weighted minimum-norm estimate (wMNE). This use of BrainStorm software package is documented, and the software itself is freely available for download online under the GNU General Public License (Tadel et al., 2011). Cortical current source density mapping was obtained from a distributed source model of 15,000 trihedral current dipoles (equivalent to sources unconstrained in their orientation) distributed over the cortical mantle of a generic brain model built from the standard "MNI/Colin27" brain template of the Montreal Neurological Institute, available in the Brainstorm distribution. This head model was co-registered with the MEG sensor locations using the fiducials (nasion, right and left auricular points) and the digitized head points thanks to an iterative alignment algorithm implemented in Brainstorm. The MEG forward model was computed with the overlapping-spheres analytical model. For each participant, a noise covariance matrix was computed on the basis of the $100-\mathrm{ms}$ baseline period prior to the initial face onset, using the single trials from which the ERFs were computed. This noise covariance matrix was taken into account in the inversion algorithm. Cortical current maps were computed from the MEG time series in response to the targets under each condition of interest for each participant. The cortical maps in response to the right and left target displays averaged across valid and invalid conditions were also calculated and transformed into z-score with respect to the mean and standard deviation of the activity during the baseline period and grand averaged across subjects for the purpose of defining the set of regions activated in response to the targets (see below).

\subsection{Behavioral data analyses}

Accuracy and reaction time (RT) in the categorization task were computed from the 6 main blocks of attention cueing. Only correct responses with RT values between 200 and $2500 \mathrm{~ms}$ were taken into account for RT analysis. The RT data were analyzed using two-way repeated-measures ANOVA with target validity (valid/invalid) and side (right/left) as within-subjects factors. 


\subsection{Source data analyses}

In order to concentrate on regions responsive to the targets and showing good signal-to-noise ratio, we first analyzed the data to create a target-specific search volume from the integral imaging data. This volume was then used to constrain analyses of the effects of the experimental conditions in an unbiased manner (see e.g. Cross et al., 2012 for a similar procedure). This procedure was achieved by examining the neural activity evoked in response to the targets relative to the baseline period. We calculated the grand average of the z-scored cortical maps in response to the right and left targets, pooling the valid and invalid conditions. We defined the regions of interest (ROIs or search volume) as the regions extending over at least 100 contiguous vertices with a cortical source activity above $65 \%$ of the maximal source amplitude of $20 \mathrm{pA}$, at the maxima of the root-mean-square (RMS) of the ERFs in response to the targets. The RMS was computed as the square root of the mean of the squared ERF amplitude across sensors at each point in time (Kenney and Keeping, 1962). Our procedure allowed us to identify bilateral ROIs in the occipital, temporal, and parietal regions, and two left-lateralized ROIs in the motor region and the insula. As these ROIs were identified from data z-scored according to the baseline period, they corresponded to the regions significantly activated by the targets. These regions were used as our search volume for the subsequent analyses.

Then, to analyze the cortical sources of target processing, we extracted the raw (non-normalized) time series of the cortical current sources (computed as the norm of the trihedral dipoles at each time point) at every vertex of the previously defined ROIs for each participant and each condition. We conducted statistical analyses on these sources. Point-by-point ANOVAs were performed at each vertex and at each time point between $100 \mathrm{~ms}$ before and $700 \mathrm{~ms}$ after target onset. We analyzed the neural responses associated with the attention orienting effect of eye gaze by examining the sources of the ERFs to valid and invalid right and left targets. For this, we performed point-by-point ANOVAs with validity (valid versus invalid) and target side (left versus right) as within-subject factors and Student $t$-tests contrasting the valid and invalid conditions for the right and left targets respectively. For all statistical analyses, we considered as significant only the regions activated during target processing, i.e. encompassed in the above defined ROIs, where at least 10 vertices reached a significance threshold of $\mathrm{p}<.005$ over at least 10 consecutive time samples.

\section{Results}

\subsection{Behavioral results}

The target items (letters and symbols) were categorized at ceiling (mean percentage of correct response \pm S.E.M $=98 \pm .5$ ). There was a significant gaze cueing effect with faster response times (RT) for validly cued (598 $\pm 21 \mathrm{~ms})$ as compared to invalidly cued targets $(608 \pm 22 \mathrm{~ms} ; \mathrm{F}(1,23)=8.22, \mathrm{p}<.01)$. There was a non-significant trend to an effect of target side $(\mathrm{F}(1,23)=3.04, \mathrm{p}=.09)$, with overall slightly faster RT to left targets $(601 \pm 21 \mathrm{~ms})$ than to right targets $(606 \pm 22 \mathrm{~ms})$. The interaction between validity and target side was not significant $(\mathrm{F}<1)$.

\section{2. $M E G$ results}

The time course of magnetic activities evoked throughout the course of the trials is depicted in the Fig. 2. We observed successive peaks of activity associated with the stimulus sequence: A first set of ERF responses was observed after the initial face onset; a second set followed the eye gaze shift; and the final set was elicited by the target display. These successive waves of activity can be best visualized on the RMS of the ERFs. We focused on the magnetic responses to the target. Peaks of magnetic activities in response to the target were observed at 130, 190,
285,400 , and $475 \mathrm{~ms}$ after the onset of the target display on the grand average of the data (Fig. 2). For illustrative purpose, Fig. 3 presents the corresponding topographical maps of the ERFs.

We chose to analyze these data going directly at the source level. The reason for this is that source localization is a linear method, performing signal deconvolution at the cortical level. Therefore, analyzing signal at the scalp level and at the source level, at least when using a common brain template as was the case here, may be seen as constitutive of double dipping (Kriegeskorte et al., 2009). We performed source localization of the ERFs in response to the target. The grand averaged cortical current maps obtained at the time instants of the maxima of magnetic responses identified on the RMS to the target are illustrated in the Fig. 4. As is classically observed (e.g. Niemeier et al., 2005), we found a retinotopic organization of the responses to the target in the occipital regions. An extensive activation of the occipital medial and lateral regions controlateral to the target was seen from about $100 \mathrm{~ms}$. It reached a maximum around $130 \mathrm{~ms}$ and then spread over temporal and parietal regions, including the posterior superior temporal sulcus (STS)/temporo-parietal junction (TPJ) region and the superior parietal lobule region. It is interesting to note that the activations in the right temporal region was observed for both right- and left-side targets. Activation in the left insular/inferior frontal region was also seen around $190 \mathrm{~ms}$ in response to the target displayed on the right side. The activations in the parietal lobule regions seemed to be left lateralized from about $285 \mathrm{~ms}$ and these left lateralized parietal activities were observed for both right- and left-side targets. Finally, a peak of activation was observed in the left motor region from about $400 \mathrm{~ms}$ (remember that all responses were given with the right hand). This set of activated regions was defined as our set of ROIs or search volume to investigate the effects of attention induced by gaze on the visual processing of targets.

We analyzed the modulation of target processing by the gaze cueing of attention. For this, we performed an ANOVA on the cortical current amplitudes at every vertex of our search volume and every time point between -100 and $+700 \mathrm{~ms}$, with target validity and side as withinsubject factors. As this involved multiple comparisons, we considered as significant only the regions encompassing at least 10 vertices that reached a significance threshold of $\mathrm{p}<.005$ over 10 or more consecutive time samples. Since we observed activities strongly lateralized to the hemisphere contralateral to the targets, particularly in the occipital regions, we also ran Student $t$-tests contrasting the valid and invalid conditions for targets appearing on the left and right sides respectively (with the same significance threshold of at least 10 vertices with $\mathrm{p}<.005$ over $>10 \mathrm{~ms}$ ). We found a main effect of validity in three posterior regions of the right hemisphere (Fig. 5). This effect reflected higher neural responses for invalid relative to valid targets in the contralateral visual field (i.e. left targets) as confirmed by Student $t$ tests. It occurred between 91 and $400 \mathrm{~ms}$, between 127 and $282 \mathrm{~ms}$, and between 208 and $228 \mathrm{~ms}$ in the posterior parietal (14 vertices), lateral occipital (50 vertices) and medial occipital regions (13 vertices) respectively. As can be seen in Fig. 5, the validity effect was not continuous in these time windows, but rather predominant in the time intervals of maximum activity in each region. The effect of validity did not reach significance for the right-side targets in the right hemisphere. Greater responses to invalid than valid targets were also found in the left hemisphere, with this effect being constrained to contralateral right-side targets, as demonstrated by the Student $t$-tests. This effect peaked in the left superior parietal lobule region between 174 and 218 ms (25 vertices; Fig. 5).

\section{Discussion}

We investigated the cerebral substrates of visual target processing and how it is modulated by attention orienting to gaze. The attention orienting effect of gaze was reflected in faster reaction times to valid than invalid targets. At the neural level, we observed an early 

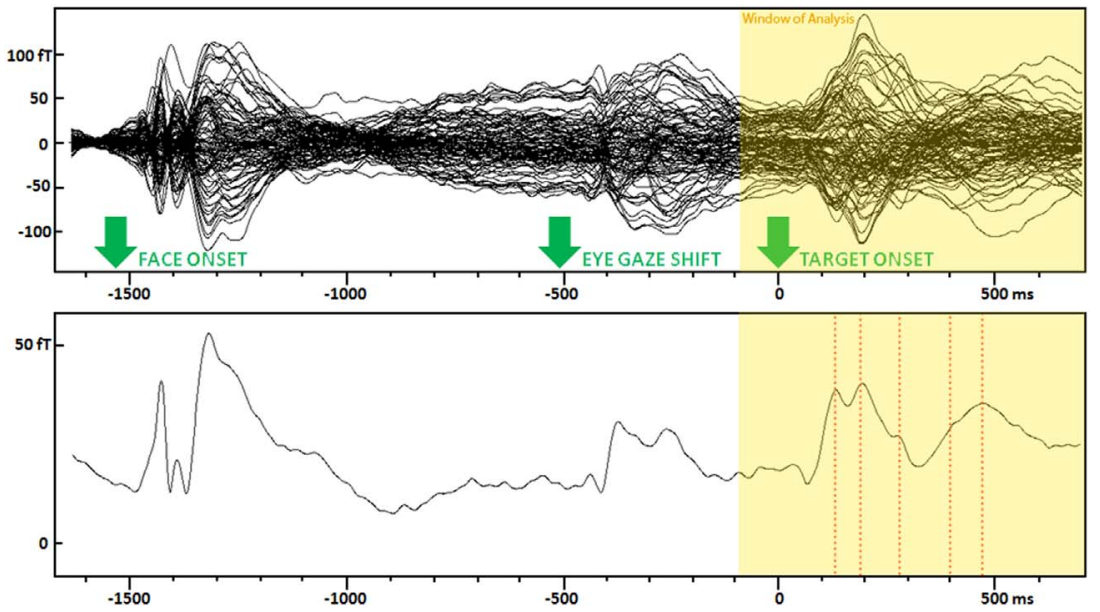

Fig. 2. Grand mean ERFs elicited along the trial sequence. Top: Timecourse of the grand-averaged event-related magnetic fields (ERFs) obtained across all conditions from $100 \mathrm{~ms}$ before the onset of the initial face to $700 \mathrm{~ms}$ after the target onset. The baseline period corresponded to the $100 \mathrm{~ms}$ preceding the initial face onset. An overlay of all magnetometers is presented. Bottom: Timecourse of the root mean square (RMS) of the grand averaged evoked magnetic activity. The RMS was computed across all magnetometers. The vertical red dotted lines highlight the peaks of magnetic activities obtained in response to the targets at $130,190,285,400$, and $475 \mathrm{~ms}$ (time zero $=$ target onset). (For interpretation of the references to color in this figure legend, the reader is referred to the web version of this article.)

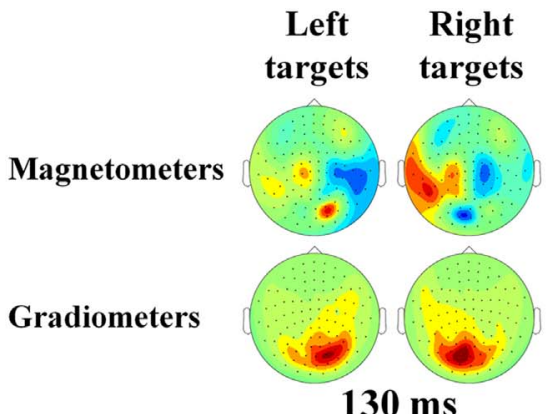

$130 \mathrm{~ms}$

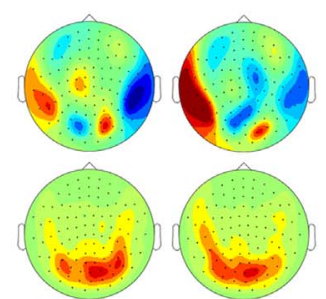

$190 \mathrm{~ms}$

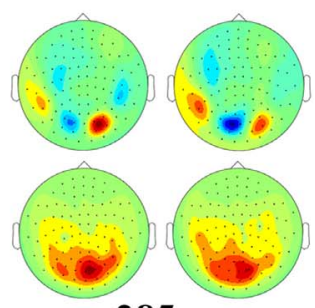

$285 \mathrm{~ms}$

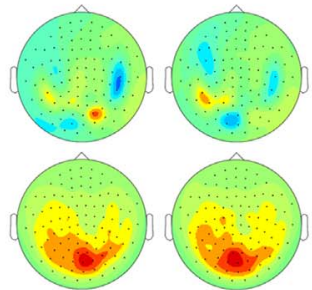

$400 \mathrm{~ms}$

Magnetometers

Gradiometers

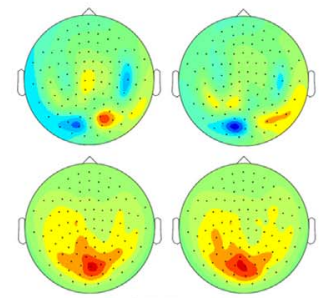

$475 \mathrm{~ms}$
Fig. 3. Maps of the grand mean magnetic fields and gradients at the maxima of the RMS to the targets. At each time instant, topographic maps of the ERF measured on the magnetometers (upper row) and of the ERF gradients computed as the norm of the two orthogonal gradiometer measures at each scalp location (lower row) are represented separately for the targets presented on the left side of the screen (Left) and on the right side of the screen (Right). (For visualisation of the colour scales in this figure, the reader is referred to the web version of this article.)

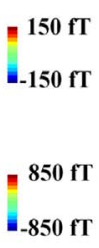

$850 \mathrm{fT}$
$-850 \mathrm{fT}$

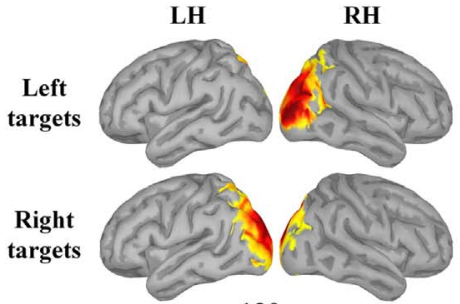

$130 \mathrm{~ms}$

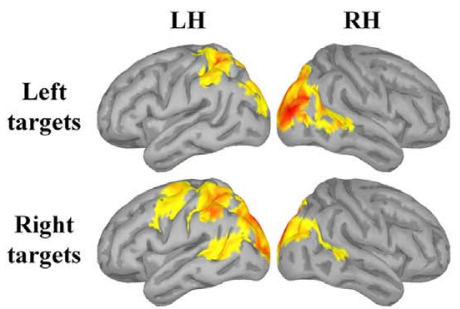

$400 \mathrm{~ms}$

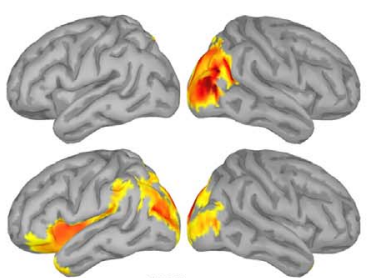

$190 \mathrm{~ms}$

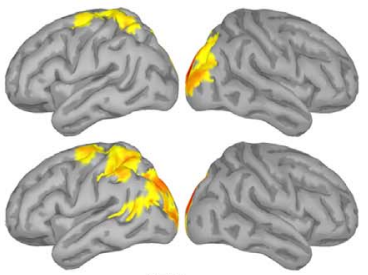

$475 \mathrm{~ms}$

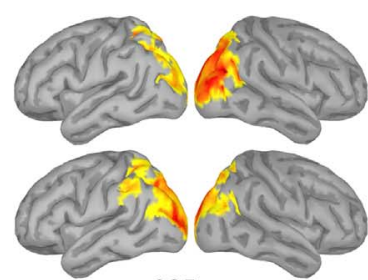

$285 \mathrm{~ms}$

Fig. 4. Grand mean cortical current maps for leftand right-side targets. At each time instant, the grand-averaged cortical current sources obtained for the targets presented on the left side (Left targets; upper row) and on the right side of the screen (Right targets; lower row) are superimposed on a left (LH) and a right (RH) lateral, inflated views of the MNI template brain. The activation of the sources is colorcoded only for the $65 \%$ maximum of activity (in zscore units). (For interpretation of the references to color in this figure legend, the reader is referred to the web version of this article.)

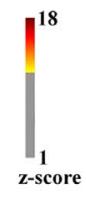

appearing on the left visual field were associated with enhanced brain responses for invalid relative to valid targets between 91 and $400 \mathrm{~ms}$ in

modulation of the brain responses to targets as a function of the congruence between target location and the location cued by gaze. This modulation was maximal contra-lateral to the target. The targets

\begin{abstract}
the right posterior parietal and occipital cortices. The targets appearing
\end{abstract}




\section{LEFT EFFECTS RIGHT EFFECTS}

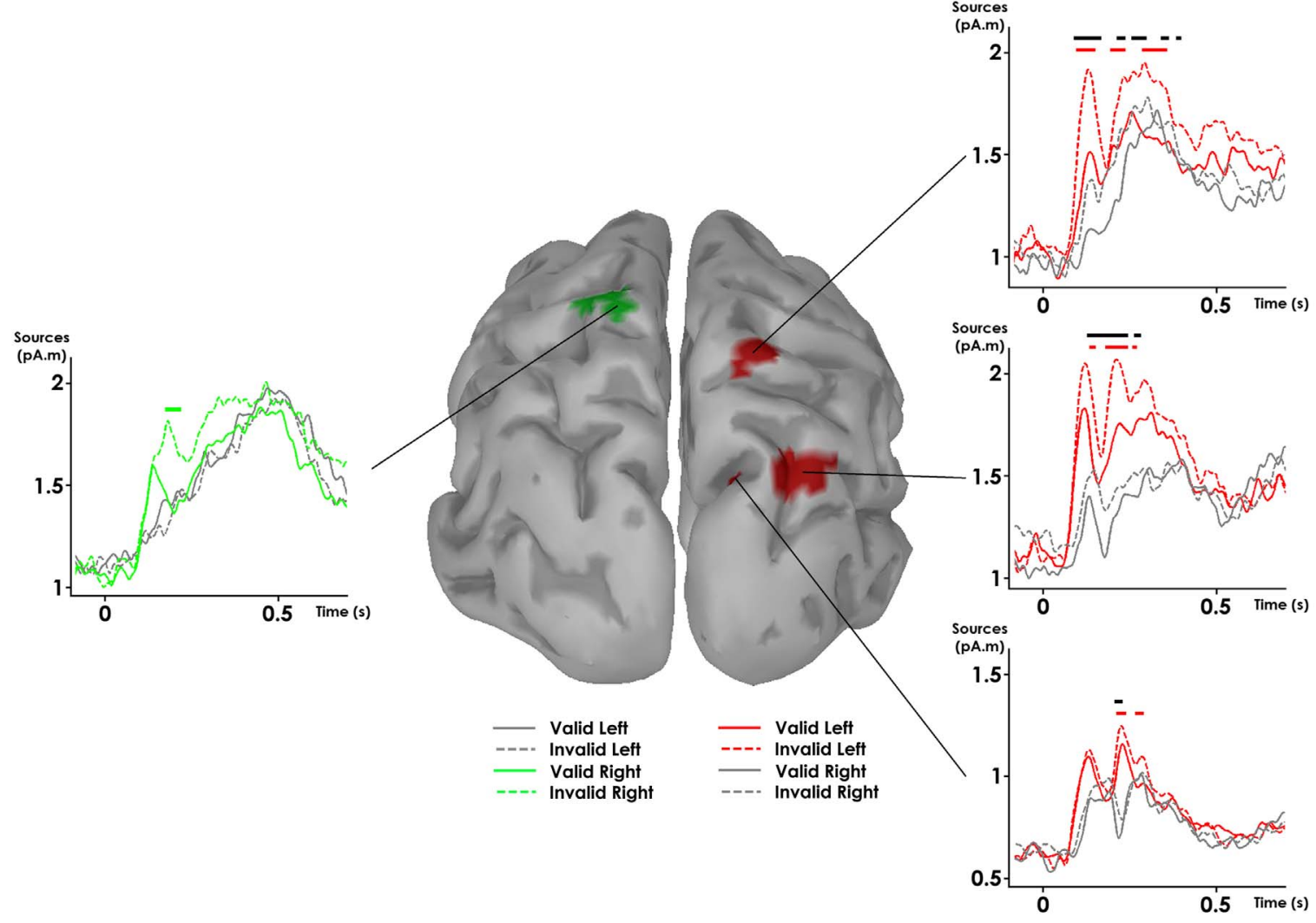

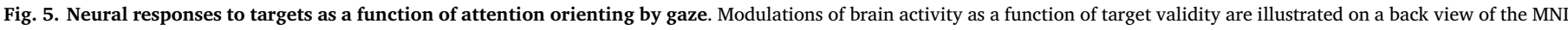

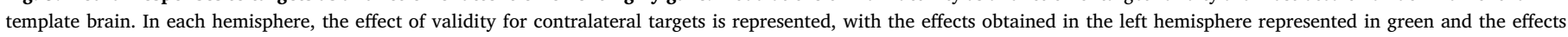

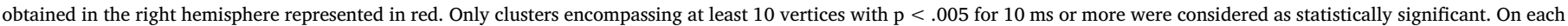

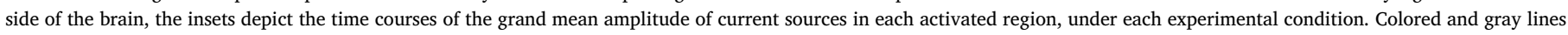

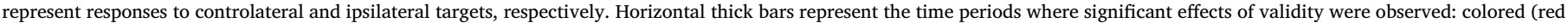

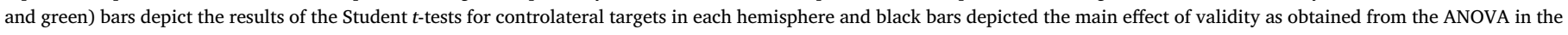
right hemisphere. (For interpretation of the references to color in this figure legend, the reader is referred to the web version of this article.)

in the right visual field were associated with enhanced responses to invalid relative to valid targets between 174 and $218 \mathrm{~ms}$ in the left superior parietal cortex.

Previous studies have shown a consistent effect of attention cueing by eye gaze in the form of faster responses for valid relative to invalid targets (e.g. Driver et al., 1999; see Frischen et al., 2007 for review). Here, we found a $10 \mathrm{~ms}$ advantage of RTs for valid relative to invalid targets, consistent with previous studies on eye gaze cueing (see Lachat et al., 2012a for a review of the literature on this effect). We thus replicate this well-established phenomenon that reflects the attention orienting power of perceived eye gaze. This behavioral effect was associated with changes in the neural responses to valid versus invalid targets. We showed modulations of the brain responses to the targets from $91 \mathrm{~ms}$ after left-target onset in the right posterior parietal cortex, extending into right occipital regions between 127 and $282 \mathrm{~ms}$. There was also a modulation of brain responses between 174 and $218 \mathrm{~ms}$ in the left superior parietal lobule region, for right targets. These results converge with previous ERP studies that have shown early amplitude modulations of the P1 (90-120 ms) and N1 (150-190 ms) components in similar gaze cueing paradigms (Schuller and Rossion, 2001, 2004, 2005). They confirm that attention orienting by gaze impacts on the early stages of the visual processing of targets.

In previous ERP studies, P1 and N1 were generally found to be greater for valid than invalid targets (Schuller and Rossion, 2004, 2005; Tipper et al., 2008; Hietanen et al., 2008; Brignani et al., 2009). This stands in contrast with our finding of greater occipito-parietal responses for invalid than valid targets. Interestingly, effects in the same direction (that is, with enhanced activation for invalid relative to valid targets) have been reported in several fMRI studies that investigated the cueing effects of attentional cues such as arrows (Engell et al., 2010) and eye gaze (Joseph et al., 2015). These authors interpreted the greater neural responses to invalid than valid targets as reflecting the reorienting processes elicited when a target appears at an unexpected location, resulting in a mismatch between the sensory input and the subject's expectation. Indeed, eye gaze is particularly powerful at inducing expectations about target location, as shown by the fact that the gaze cueing effect has been observed even with counter-predictive gaze cues (that pointed most frequently to the side of the screen opposite to the target; Driver et al., 1999). This view is also supported by the fMRI studies that investigated the role of the posterior superior temporal sulcus (pSTS) in the coding of gaze direction (e.g. Pelphrey et al., 2003; Wyk et al., 2009, see also Carlin and Calder, 2013 for a review) and demonstrated enhanced pSTS activation in situation of incongruency (relative to congruency) between target location and seen gaze shifts. These studies provide evidence that eye gaze is not only a directional cue; it also conveys information about intentions and might evoke expectations regarding subsequent actions (Pelphrey et al., 2003, 2004). Accordingly, Joseph et al. (2015) suggested that while non social cues such as arrows may be very powerful at engaging orienting processes visible in amplified responses to valid relative to invalid targets, gaze would be very potent at eliciting expectations hence inducing more robust reorienting processes, visible in increased activities to invalid relative to valid targets. Our results are in line with this view. They further demonstrate that the reorienting effects associated with 
attention cueing by gaze occur in the early stages of the visual processing of targets. Moreover, although MEG has a limited spatial resolution relative to fMRI, the right posterior parietal and lateral occipital activities and the left superior parietal activities that we revealed are consistent with the regional activations that were found for the effects of orienting by eye gaze in Joseph et al. (2015). We noted also that the modulation of the brain responses to the targets was more marked in the right than the left hemisphere. First, we found a main effect of target validity in the right hemisphere; second, the effect started earlier and was more sustained in the right than the left hemisphere, also involving a more extended set of regions. These brain modulations also concurred with a slight but non-significant trend to an RT advantage for targets located in the left relative to the right of the screen. The right hemisphere lateralization of the attention orienting effect of gaze is consistent with the dominance of the right hemisphere in attention-related processes (Mapstone et al., 2003; Foxe et al., 2003) and in particular in the attention reorienting system (Corbetta and Shulman, 2002; Corbetta et al., 2008). In sum, our finding of enhanced neural responses to invalid relative to valid targets from $\sim 90 \mathrm{~ms}$ provides evidence for the attention orienting power of gaze, associated with the early involvement of posterior attention networks. It will be interesting in future studies to disentangle what pertains to attention orienting and engagement processes versus disengagement and reorienting processes in the impact of other's gaze on surrounding object processing.

The time range and the source localization of our attention orienting effects differ substantially from the findings of the previous MEG study that was performed on the same topic. Nagata et al. (2012) found an attention orienting effect of gaze cues only in a relatively late time window (286-306 ms) and this effect involved the anterior cingulate cortex. In this study, the participants were asked to indicate the right or left location of the target, which encompassed an explicit spatial component. It is therefore possible that the activation observed by these authors reflected the processing of the cognitive conflict between attention direction (as cued by gaze) and invalid target location. It is also possible that their effect reflected a different type of attention process, because they observed an inhibition-of-return effect (viz. slower RT to valid than invalid targets)—albeit not significant for the gaze cues-rather than a gaze cueing effect. In contrast, our results seem to reflect earlier attention-related processes and target processing demands implemented in posterior parietal and occipital regions. This is consistent with the previous study by Lachat et al. (2012b), which demonstrated early engagement of occipito-parietal cortices associated with eye gaze cueing, but using fearful face stimuli. The use of emotional faces may explain why an earlier effect was found in this previous paper. It is however also to be noted that this earlier effect was left lateralized and in the form of greater activity for valid than invalid targets. This suggests that it reflected a different type of process, possibly dominated by fast orienting processes related to emotional gaze, while our effect seemed rather related to early reorienting processes associated with attention cueing by neutral gaze. Thus, we provide the first neuromagnetic evidence for the influence of attention orienting by neutral face gaze on the early stages of target processing.

In summary, this study showed that the effect of attention orienting by eye gaze cues is associated with early modulation of target processing - from $90 \mathrm{~ms}$ post-target onset, involving posterior parietal and occipital cortices. This modulation seemed to reflect the additional processing demand associated with attention reorienting for invalid relative to valid targets. These findings contribute to better characterize how visual processing is modulated by social information, which is key to further advance the understanding of the influence that others can have on our perception of the world.

\section{Acknowledgments}

We thank Christophe Gitton, Antoine Ducorps, Laurent Hugueville, and Yohan Attal for their assistance in data acquisition and pre-processing. We also thank anonymous reviewers whose comments allowed to improve the quality of the manuscript. This work was supported by the French program "Investissement d'Avenir" under grant numbers ANR-10-IAIHU-06 and ANR-11-INBS-0006, by Grants from the Agence Nationale de la Recherche to SD and NG (projects "SENSO" and "AVE"), and by Grants from the Comisión Nacional de Investigación Científica y Tecnológica (CONICYT, Chile, PCHA, Master 2y Doctorado), from the Fondation de France (Comité Berthe Fouassier; Grant no. 201200029844), and from a partial support by Millennium Nucleus Biology of Neuropsychiatric Disorders Nu-MIND, ICM-MINECOM Grant NC 130011 to JLU. None of these funding bodies had a role in study design, in the collection, analysis and interpretation of data, in the writing of the report, and in the decision to submit the article for publication.

\section{References}

Bayless, S.J., Nagata, Y., Mills, T., Taylor, M.J., 2013. MEG measures of covert orienting and gaze processing in children. Brain Topogr. 26, 616-626.

Bayliss, A.P., Paul, M.A., Cannon, P.R., Tipper, S.P., 2006. Gaze cuing and affective judgments of objects: I like what you look at. Psychon. Bull. Rev. 13, 1061-1066.

Becchio, C., Bertone, C., Castiello, U., 2008. How the gaze of others influences object processing. Trends Cogn. Sci. 12, 254-258.

Brignani, D., Guzzon, D., Marzi, C.A., Miniussi, C., 2009. Attentional orienting induced by arrows and eye-gaze compared with an endogenous cue. Neuropsychologia 47, 370-381.

Carlin, J.D., Calder, A.J., 2013. The neural basis of eye gaze processing. Curr. Opin. Neurobiol. 23, 450-455.

Corbetta, M., Shulman, G.L., 2002. Control of goal-directed and stimulus-driven attention in the brain. Nat. Rev. Neurosci. 3, 201-215.

Corbetta, M., Patel, G., Shulman, G.L., 2008. The reorienting system of the human brain: from environment to theory of mind. Neuron 58, 306-324.

Cross, E.S., Cohen, N.R., Hamilton, A.F. de C., Ramsey, R., Wolford, G., Grafton, S.T., 2012. Physical experience leads to enhanced object perception in parietal cortex: insights from knot tying. Neuropsychologia 50, 3207-3217.

Driver, J., Davis, G., Ricciardelli, P., Kidd, P., Maxwell, E., Baron-Cohen, S., 1999. Gaze perception triggers reflexive visuospatial orienting. Vis. Cogn. 6, 509-540.

Engell, A.D., Nummenmaa, L., Oosterhof, N.N., Henson, R.N., Haxby, J.V., Calder, A.J., 2010. Differential activation of frontoparietal attention networks by social and symbolic spatial cues. Soc. Cogn. Affect. Neurosci. 5, 432-440.

Foxe, J.J., McCourt, M.E., Javitt, D.C., 2003. Right hemisphere control of visuospatial attention: line-bisection judgments evaluated with high-density electrical mapping and source analysis. NeuroImage 19, 710-726.

Friesen, C.K., Kingstone, A., 1998. The eyes have it! Reflexive orienting is triggered by nonpredictive gaze. Psychon. Bull. Rev. 5, 490-495.

Frischen, A., Bayliss, A.P., Tipper, S.P., 2007. Gaze cueing of attention: visual attention, social cognition, and individual differences. Psychol. Bull. 133, 694-724.

Hietanen, J.K., Nummenmaa, L., Nyman, M.J., Parkkola, R., Hämäläinen, H., 2006. Automatic attention orienting by social and symbolic cues activates different neural networks: an fMRI study. NeuroImage 33, 406-413.

Hietanen, J.K., Leppänen, J.M., Nummenmaa, L., Astikainen, P., 2008. Visuospatial attention shifts by gaze and arrow cues: an ERP study. Brain Res. 1215, 123-136.

Itier, R.J., Batty, M., 2009. Neural bases of eye and gaze processing: the core of social cognition. Neurosci. Biobehav. Rev. 33, 843-863.

Joseph, R.M., Fricker, Z., Keehn, B., 2015. Activation of frontoparietal attention networks by non-predictive gaze and arrow cues. Soc. Cogn. Affect. Neurosci. 10, 294-301.

Kriegeskorte, N., Simmons, W.K., Bellgowan, P.S.F., Baker, C.I., 2009. Circular analysis in systems neuroscience: the dangers of double dipping. Nat. Neurosci. 12, 535-540.

Kenney, J.F., Keeping, E.S., 1962. In Mathematics of Statistics, Pt. 1. In: Princeton, N.J, Nostrand, Van (Eds.), Root Mean Square. §4.15, 3rd ed. pp. 59-60.

Lachat, F., Conty, L., Hugueville, L., George, N., 2012a. Gaze cueing effect in a face-toface situation. J. Nonverbal Behav. 36, 177-190.

Lachat, F., Farroni, T., George, N., 2012b. Watch out! Magnetoencephalographic evidence for early modulation of attention orienting by fearful gaze cueing. PLoS One 7, e50499.

Mapstone, M., Weintraub, S., Nowinski, C., Kaptanoglu, G., Gitelman, D.R., Mesulam, M.M., 2003. Cerebral hemispheric specialization for spatial attention: spatial distribution of search-related eye fixations in the absence of neglect. Neuropsychologia 41, 1396-1409.

Nagata, Y., Bayless, S.J., Mills, T., Taylor, M.J., 2012. Spatio-temporal localisation of attentional orienting to gaze and peripheral cues. Brain Res. 1439, 44-53.

Niemeier, M., Goltz, H.C., Kuchinad, A., Tweed, D.B., Vilis, T., 2005. A contralateral preference in the lateral occipital area: sensory and attentional mechanisms. Cereb. Cortex 15, 325-331.

Nummenmaa, L., Calder, A.J., 2009. Neural mechanisms of social attention. Trends Cogn. Sci. 13, 135-143.

Pelphrey, K.A., Singerman, J.D., Allison, T., McCarthy, G., 2003. Brain activation evoked by perception of gaze shifts: the influence of context. Neuropsychologia 41, 156-170.

Pelphrey, K.A., Viola, R.J., McCarthy, G., 2004. When strangers pass: processing of mutual and averted social gaze in the superior temporal sulcus. Psychol. Sci. 15, 
598-603.

Posner, M.I., 1980. Orienting of attention. Q. J. Exp. Psychol. 32, 3-25.

Sato, W., Kochiyama, T., Uono, S., Yoshikawa, S., 2009. Commonalities in the neural mechanisms underlying automatic attentional shifts by gaze, gestures, and symbols. NeuroImage 45, 984-992.

Schuller, A.-M., Rossion, B., 2001. Spatial attention triggered by eye gaze increases and speeds up early visual activity. Neuroreport 12, 2381-2386.

Schuller, A.-M., Rossion, B., 2004. Perception of static eye gaze direction facilitates subsequent early visual processing. Clin. Neurophysiol. 115, 1161-1168.

Schuller, A.-M., Rossion, B., 2005. Spatial attention triggered by eye gaze enhances and speeds up visual processing in upper and lower visual fields beyond early striate visual processing. Clin. Neurophysiol. 116, 2565-2576.

Tadel, F., Baillet, S., Mosher, J.C., Pantazis, D., Leahy, R.M., 2011. Brainstorm: a userfriendly application for MEG/EEG analysis. Comput. Intell. Neurosci. 2011, e879716. Tipper, C.M., Handy, T.C., Giesbrecht, B., Kingstone, A., 2008. Brain responses to biological relevance. J. Cogn. Neurosci. 20, 879-891.

Ulloa, J.L., Marchetti, C., Taffou, M., George, N., 2014. Only your eyes tell me what you like: exploring the liking effect induced by other's gaze. Cogn. Emot. 23, 1-11.

Wyk, B.C.V., Hudac, C.M., Carter, E.J., Sobel, D.M., Pelphrey, K.A., 2009. Action understanding in the superior temporal sulcus region. Psychol. Sci. 20, 771-777. 